\title{
231509
}

UCRL-JC-127158

PREPRINT

\section{Single Scatter Electron Monte Carlo}

\author{
Michelle M. Svatos \\ Lawrence Livermore National Laboratory \\ Livermore, $\mathrm{CA}$ \\ and \\ Univeristy of Wisconson - Madison \\ Madison, WI
}

This paper was prepared for submittal to the

Joint International Conference on Mathematical Methods and

Supercomputing for Nuclear Applications

Saratoga Springs, NY

October 6-10. 1997

March, 1997

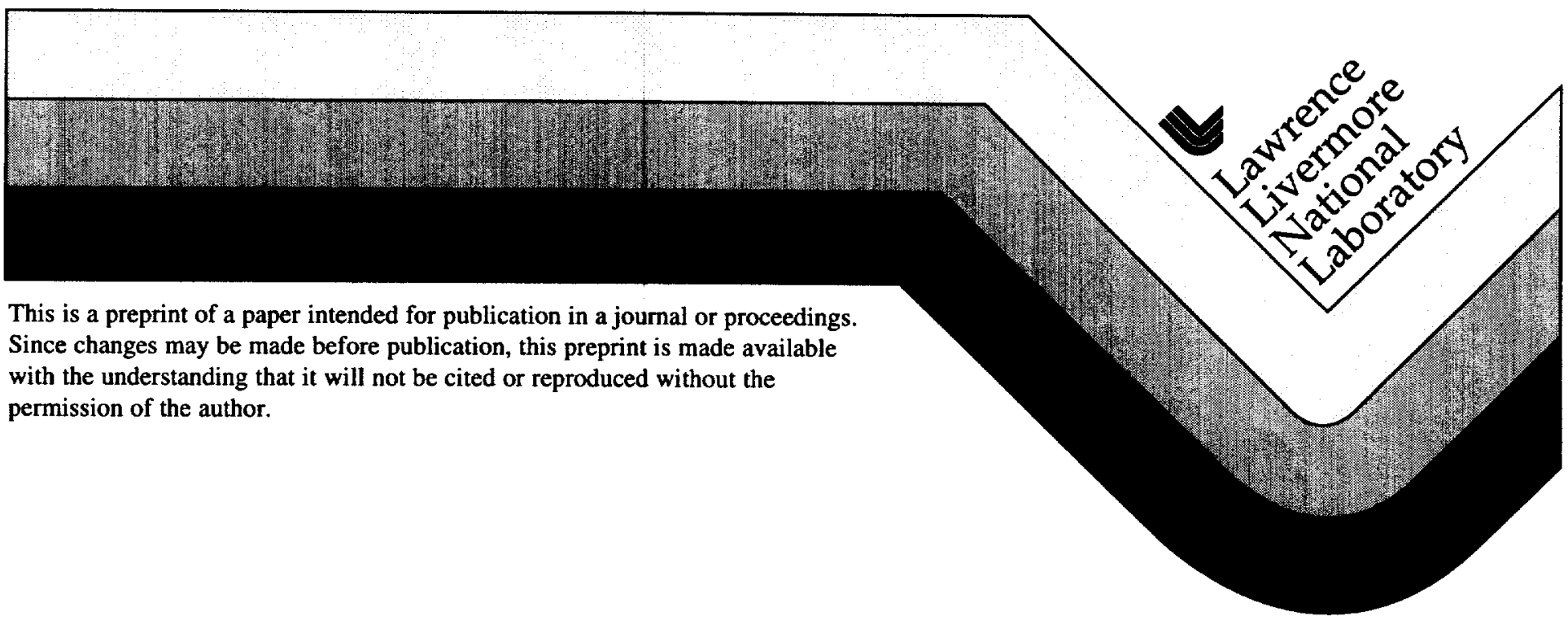




\section{DISCLAIMER}

This document was prepared as an account of work sponsored by an agency of the United States Government. Neither the United States Government nor the University of California nor any of their employees, makes any warranty, express or implied, or assumes any legal liability or responsibility for the accuracy, completeness, or usefulness of any information, apparatus, product, or process

disclosed, or represents that its use would not infringe privately owned rights. Reference herein to any specific commercial product, process, or service by trade name, trademark, manufacturer, or otherwise, does not necessarily constitute or imply its endorsement, recommendation, or favoring by the United States Government or the University of California. The views and opinions of authors expressed herein do not necessarily state or reflect those of the United States Government or the University of California, and shall not be used for advertising or product endorsement purposes. 


\title{
SINGLE SCATTER ELECTRON MONTE CARLO
}

\author{
M.M. Svatos
}

\author{
Lawrence Livermore National Laboratory \\ 7000 East A venue \\ Livermore, CA 94550 \\ svatos@l!nl.gov
}

\author{
University of Wisconsin-Madison \\ 1300 University A venue \\ Madison, WI 53706 \\ svatos@madrad.radiology.wisc.edu
}

\begin{abstract}
A single scatter electron Monte Carlo code (SSMC), CREEP, has been written which bridges the gap between existing transport methods and modeling real physical processes. CREEP simulates ionization, elastic and bremsstrahlung events individually. Excitation events are treated with an excitation-only stopping power. The detailed nature of these simulations allows for calculaticn of backscatter and transmission coefficients, backscattered energy spectra, stopping powers, energy deposits, depth dose, and a variety of other associated quantities. Although computationally intense, the code relies on relatively few mathematical assumptions, unlike other charged particle Monte Carlo methods such as the commonly-used condensed history method. CREEP relies on sampling the Lawrence Livermore Evaluated Electron Data Library (EEDL) which has data for all elements with an atomic number between 1 and 100, over an energy range from approximately several $\mathrm{eV}$ (or the binding energy of the material) to $100 \mathrm{GeV}$. Compounds and mixtures may also be used by combining the appropriate element data via Bragg additivity.
\end{abstract}

\section{INTRODUCTION}

Simulating electron interactions in media involves unique challenges relative to other types of particle transport, for several reasons. First, the cross sections for electrons tend to be five or six orders of magnitude higher than those for neutrons or photons of the same energy, so simulation of each collision has historically been too time-consuming to be done routinely. For instance, the mean free path of a $1 \mathrm{MeV}$ electron in water is approximately $10^{-4} \mathrm{~cm}$, which is quite small in relation to the volumes of interest for most transport problems.

Secondly, the fractional energy loss per collision is frequently five orders of magnitude smaller for electrons than it is for photons. This indicates that the number of interactions required for an electron to give up all of its energy is very large. With a few rare but important exceptions, single interactions do not change the electron's phase space by a large amount. It is rather the cumulative effect of many small changes that result in the characteristics of the electron track. Thus it is important to have accurate physics for each event, since errors will easily compound as the electron slows down.

Finally, even though most of the changes in energy and angle are small, they are not insignificant. For heavier charged particles, one can usually make a simple (Gaussian-like) approximation for the lateral distribution; for electrons, these approximations do not hold as well because they tend to underestimate the degree of lateral transport.

\section{A. Why Single Scatter?}

Single scatter physics is gaining attention for electron transport, despite the fact that it is inherently very time consuming. One reason is that since single scatter calculations conform (more) closely to the physical processes the electron undergoes, they can serve as a means to explore the validity of assumptions 
used in other transport techniques. In particular, single scattering can help make efficient electron transport methods, like condensed history, ${ }^{1}$ more accurate.

SSMC allows large angle scatter and backscatter measurements to be calculated with greater accuracy in a reliable manner. Large angle scatter and backscatter, being relatively rare, result in much of the seemingly eccentric energy deposition behavior of electron beams (and photon beams for that matter, since photons deposit their energy to the medium through secondary electrons), including lateral blooming with distance and nonuniformities ("hot" or "cold" spots) found near changes in the medium type or density.

\section{B. The EEDL Database}

The Evaluated Electron Data Library was established at LLNL by 1990 to complement the ENDL (Evaluated Nuclear Data Library) and EPDL (Evaluated Photon Data Library). Complete documents detailing its contents, with derivations, are available [1-3]. Cross sections for each atomic subshell, for each interaction, are tabulated on an energy grid with a variable placement of points between $10 \mathrm{eV}$ and $100 \mathrm{GeV}$, for atomic numbers 1 to 100 .

The elastic scattering cross sections are based on those of Mott for energies greater than $256 \mathrm{keV}$ and of Riley below $256 \mathrm{keV}$. These data were then extrapolated to cover the entire energy range. Spectra, in the form of probability distribution functions (PDFs), of angular deflections for a variety of incident energies are also tabulated. The impact ionization cross sections are based on the Moller formalism with other corrections to accurately model small energy loss collisions. Energy loss spectra are available at a number of incident energies for individual ionization and bremsstrahlung events, as well as the spectral average energy loss. The excitation database contains cross sections and the average energy loss to excitation as a function of incident energy. There are no spectral data for excitation energy loss in EEDL.

It should by noted that an independent, previous electron single scatter code, also based on the EEDL data, was written by Ballinger [4] in 1991 at LLNL and provided a strong foundation for this work.

\section{Methods}

The CREEP code is written in FORTRAN and $C$, in a very simple style with the intent of being extremely portable. Since this code is intended primarily as a means to explore basic physical properties of the medium, the present incarnation assumes only simple geometries: either spherical (user specifies radius) or slab (user specifies $x, y, z$ ), cunsisting of one type of material. Several slabs may be pieced together to simulate a layered geometry, since the output of one slab may be used as spectral input into a distal slab, and the backscattered energy spectrum from the each interface can be transported backwards in the prior medium.

\section{A. Physics Algorithms}

The overall algorithm for a truly single scatter charged particle code is a direct analog of the algorithm that has historically been used in photon and neutron Monte Carlo codes. Briefly, one finds the distance to interaction by finding the total cross section at the present energy and uses the relation $s=-\lambda \ln (\eta)$, where $\eta$ is a random number on the interval $(0,1]$. One then determines which interaction took place, by forming and sampling from a cumulative probability based on the cross sections for each of the four possible interactions (ionization, excitation, elastic scatter, bremsstrahlung). The energy, position and trajectory of the particle is updated to reflect the chosen interaction in a manner described for each below. Then the same process is begun again, provided the electron has not escaped the medium or fallen below the energy cutoff.

The CREEP code deviates from the ideal single scatter algorithm in that it does not simulate every excitation event individually. Instead, it subtracts off the expected excitation loss after each of the other

\footnotetext{
${ }^{1}$ See Appendix on Condensed History Monte Carlo for a brief explanation of this method.
} 
events, as described in the excitation section below. This choice was made because it was felt that there was little to gain by direct simulation of excitations, since they do not have a large effect on the electron trajectory, and the amount of the overall energy loss to this mechanism - although it can be large - doesn't vary much. Furthermore, since the excitation cross section is large, much simulation time would be devoted to this very small gain in accuracy.

\section{Ionization}

To simulate an ionization interaction, the knock-on electron energy is sampled from a spectrum. The EEDL database has a number of spectra tallied for various incident energies; statistical interpolation is used to choose between them. Once the energy of the knock-on has been selected, 2-body kinematics (neglecting binding energy) are used to update the primary electron's trajectory. If $T_{0}$ is the kinetic energy of the electron in electron mass units, and the ratio $\chi$ is defined by $\chi=\frac{T_{\text {snockin }}}{T_{0}}$, then the outgoing angles are given by

$$
\cos \theta_{p r i}=\sqrt{\frac{\chi\left(T_{0}+2\right)}{\left(\chi T_{0}\right)+2}}
$$

and for the knock-on electron

$$
\cos \theta_{\text {knock }}=\sqrt{\frac{(1-\chi)\left(T_{0}+2\right)}{\left((1-\chi) T_{0}\right)+2}},
$$

Note that because the binding energy is neglected, these angles are less valid at energies near it. Since such electrons don't travel far, this assumption does not have a large impact on the overall transport results.

CREEP handles secondary electrons by putting the primary on a memory "stack" and tracking the knockon immediately, until they fall below the energy cutoff or escape the volume, at which point the primary history is continued. A special energy cutoff parameter is used for knock-ons, so the user can readily imitate class II condensed history codes, which only simulate ionization events if the knock-on is above a particular energy.

Presently it is assumed that all binding energy is locally deposited. However, this assumption is known to be somewhat weak since significant fractions of the binding energy may be re-emitted as Auger electrons or fluorescent $x$-rays. While the Auger electrons do not travel far, many of the $x$-rays arc emitted at energies that fall just below the photoelectric edges, where the cross-sections are small and they can therefore carry their energy relatively far from the interaction site. Another LLNL database, EADL (Evaluated Atomic Data Library) contains all the necessary information to model these relaxations physically, but is not incorporated into the code at this time.

\section{Elastic Scattering}

In elastic scatter interactions, an incident electron traveling in the vicinity of a nucleus scatters off the nucleus at some angle without a significant loss in energy, due to the large mass difference between the two. To simulate this event, one samples a scattered angle from EEDL (where it is tabulated as $1-\mu ; \mu$ being the cosine of the scattered angle) and updates the trajectory. Although most elastic scattering results in only a small angle, it is this mechanism that is almost exclusively responsible for the phenomena of backscatter and large angle scatter.

\section{Bremsstrahlung}

Bremsstrahlung interactions occur when the electron passes near the nucleus and accelerates due to the interaction of their Coulomb fields, causing a photon to be emitted. Although low energy photons are more 
likely, an electron can lose up to all of its energy to the photon. In CREEP, the photon energy is sampled from a spectrum, and an empirical relation can be used to determine the angle of the electron after interaction:

$$
\mu=1-\left(\frac{m_{0} c^{2}}{K_{0}-h \nu}-\frac{m_{0} c^{2}}{K_{0}}\right) \text {. }
$$

The "birth" angle of the bremsstrahlung photon is more difficult; it is most correctly obtained by sampling the Schiff formula [5], but CREEP uses the approximation

$$
\theta=\frac{m_{0} c^{2}}{m_{0} c^{2}+K_{0}}
$$

where the denominator represents the total energy of the electron before the event.

Currently CREEP does not track the bremsstrahlung photons that are created; they are assumed to escape the geometry - they are effectively "lost" from the problem. This includes a loss of any additional electrons the brem photons would have generated, so CREEP cannot assume any energy deposits arising from photons. A better approach would involve storing their phase space parameters on a stack or tallied in distributions for later simulation by a photon transport code.

\section{Excitation}

The energy loss due to excitations is accounted for by finding the total excitation cross section at current energy, and using the mean energy lost to excitation events at that energy to construct an excitation-only stopping power through the relation

$$
\left(\frac{d E}{d x}\right)_{e x}=\frac{N_{A}}{A} \rho \sigma_{e x} \overline{\epsilon_{e x}}
$$

where $N_{A}$ is Avogadro's number, $A$ is the atomic weight, $\rho$ is the density, $\sigma_{e x}$ is the total excitation cross section (summed over all subshells), and $\overline{\epsilon_{e x}}$ is the mean energy loss due to excitation at a given primary energy. This stopping power is multiplied by the distance between the last event and the present event to get the energy lost to excitations in transit, which is subtracted from the electron's energy before calculating the distance to the next event.

\section{B. Compounds and Mixtures}

A special version of the CREEP code handles all compounds and mixtures by combining the EEDL element data using Bragg additivity. The user must enter mass fractions of each element in the compound or mixture. The density used is that for the compound as a whole.

This simple approach does not account for any chemical binding effects, which start to become important near the binding energy of the medium.

The algorithm is the same as that described in the beginning of the previous discussion for elements, except that once the distance to the next interaction is found, the next step is to select which medium the electron will interact with (by comparing a random number to their mass-fraction weighted cross sections) and then selecting the type of interaction as usual within that medium.

\section{Results}

Benchmarking of this code with experiment for a variety of elements and select compounds and mixtures, over the energy range of the EEDL database, is a large effort that is still in its infancy. There are many possible outputs of this code to be analyzed; some of which can be readily compared to experiments and theoretical models. Other information has no feasible experimental equivalent and as such is of interest primarily in a qualitative sense (such as "event maps" which plot interaction sites for all types of interactions). A few examples of both quantitative and qualitative results are shown in the remainder of this section. 


\section{A. Backscatter Studies}

Historically, backscatter has been difficult for condensed history codes to simulate correctly. Figure 1 shows two examples of backscatter information generated by CREEP compared to experimental values. The agreement is generally quite good.
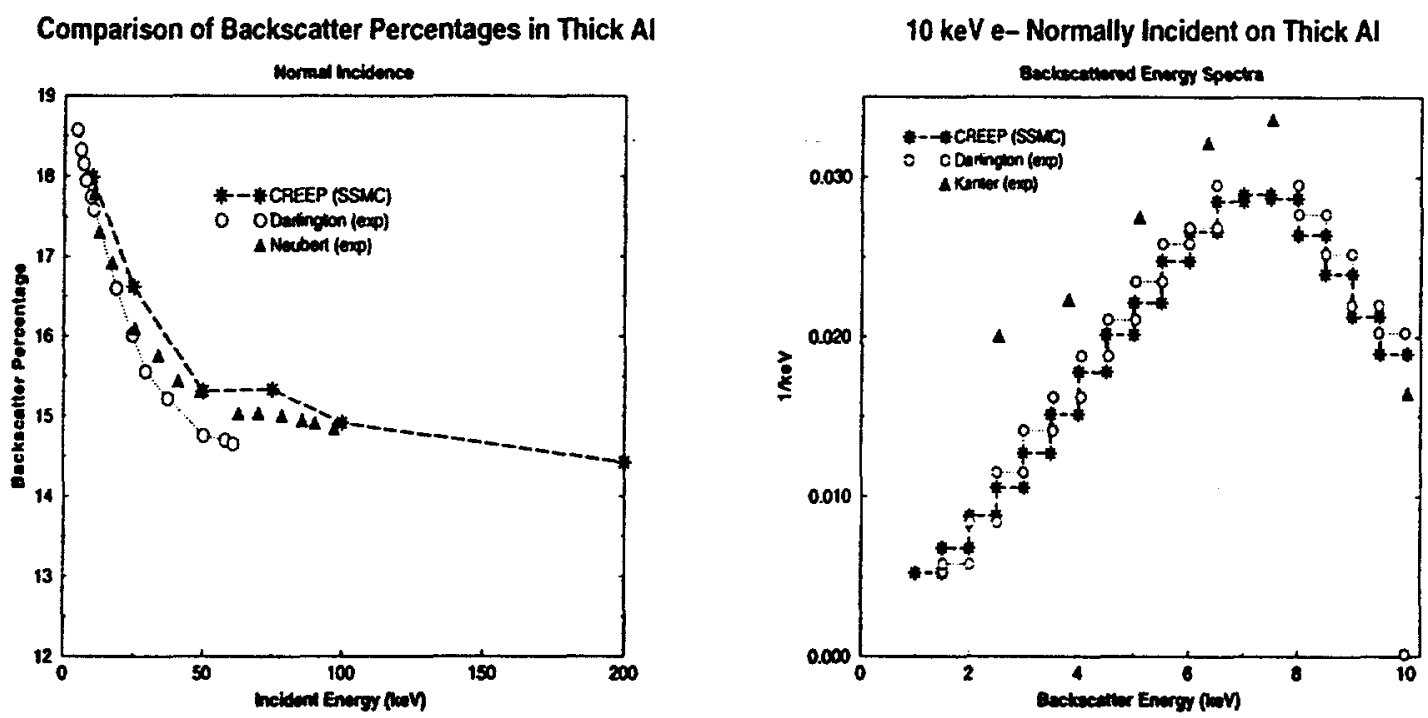

Figure 1: LEFT: CREEP backscatter percentage (including backscattered secondary electrons) compared to the experiments of Darlington et al [6] and Neubert et al [7]. RIGHT: The backscattered energy spectrum resulting from a $10 \mathrm{keV}$ electron impinging on the surface of an aluminum slab that is large in $x, y$, and $z$ compared to the mean free path of the incident electron.

\section{B. Transmission Studies}

Figure 2 strikingly illustrates both the strengths and the limitations of the present version of CREEP. For each of the four curves shown, an electron of relatively low energy (not more than 500 times the binding energy) is incident on a thin slab (not more than 20 mean free paths) and the amount of energy each electron lost after having traversed the slab is tallied. Although a Landau energy loss distribution [8] (the basis for energy loss in some condensed history codes) would predict a wide, smooth distribution, SSMC gives a highly structured, asymmetric distribution, having the same mean.

The first distinctive feature of these curves is a zero-amplitude region in the low energy loss region, implying that no electrons escape without losing at least some energy. This region ends abruptly at the energy loss that corresponds to the excitation-only stopping power times the thickness of the slab, where a sharp peak is seen. The peaks are due to electrons that escape the slab without undergoing any ionization (or brem) events. The sharpness of these peaks is therefore an artifact resulting from not modeling individual excitation events.

The next distinctive feature of these curves is a gap of low amplitude following the peak. This discontinuity is due to the binding energy of the material, which, for gold $(Z=i 9)$ is $8.3 \mathrm{eV}$ and for tantalum $(\mathrm{Z}=73)$ is $\bar{\tau} .31 \mathrm{eV}$. If an electron doesn't escape without ionization, it must give up the binding energy (in this code locally deposited in the medium), which explains the lack of electrons seen until the low, broad peak. The 

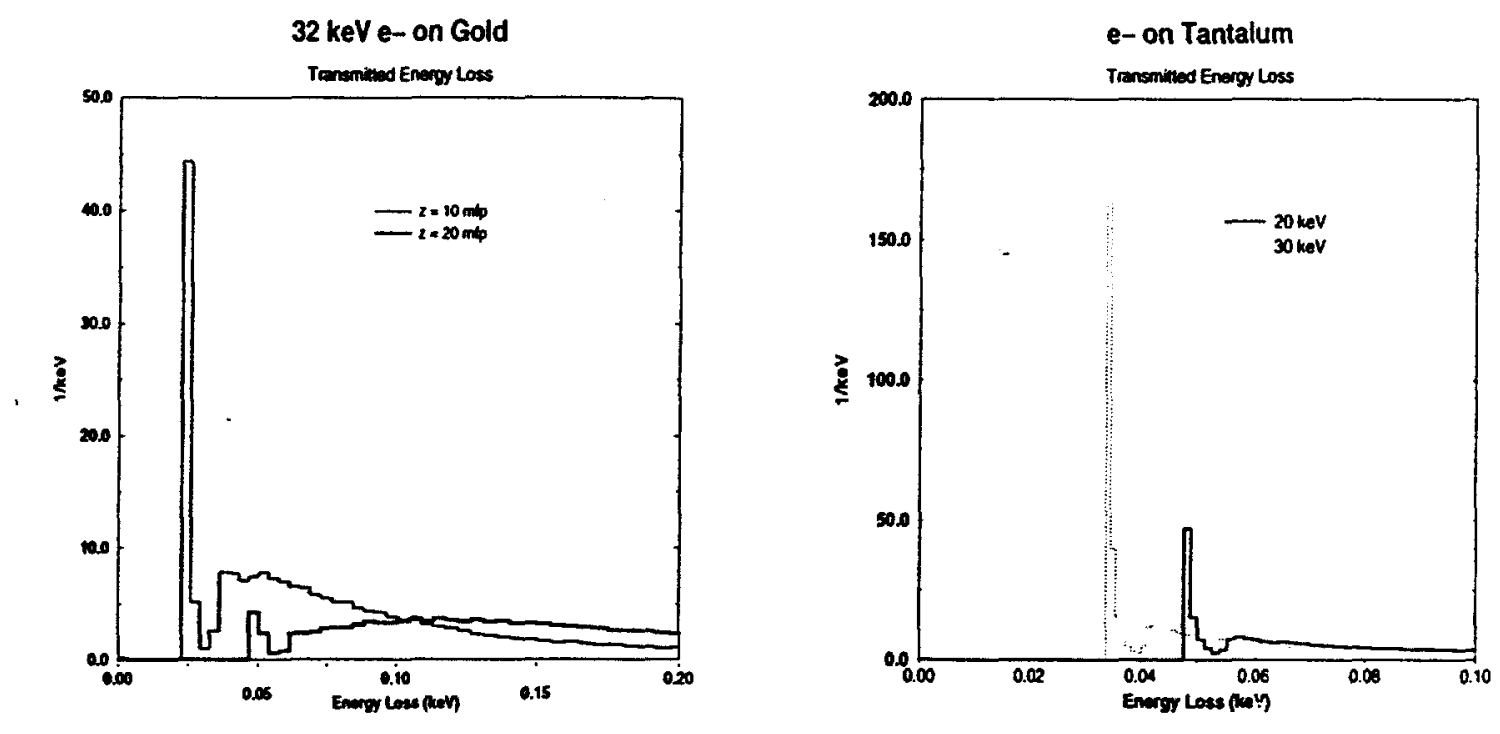

Figure 2: LEFT: The exiting energy loss spectra after $32 \mathrm{keV}$ electrons are transmitted through gold $(\mathrm{Z}=79)$ slabs of two different thicknesses: $2.87 \times 10^{-6} \mathrm{~cm}$ (approximately 10 mean free paths) and $5.74 \times 10^{-6} \mathrm{~cm}$ (approximately 20 mean free paths). RIGHT: Same in tantalum $(\mathrm{Z}=73)$ for a constant thickness of $3.0 \mathrm{x}$ $10^{-6} \mathrm{~cm}$ at two different energies. See text for discussion.

shoulder on the large-energy-loss side of this peak rolls off very gradually, as there is progressively smaller probability of multiple ionization events and/or single large energy loss events.

\section{Energy Deposition Curves}

Comparisons of the CREEP single scatter Monte Carlo (SSMC) code with experiment and with ETRAN, a class I condensed history Monte Carlo (CHMC) code, are shown in figure 3. Agreement to experiment is generally reasonable. There are some discrepancies in the build-up region (and in the peak region for aluminum) that are apparent in the comparisions between CREEP and ETRAN. These are currently under investigation, and are possibly due to the choice of interpolation routines in CREEP.

\section{Other Quantities of Interest}

In addition to the preceding quantities, CREEP also calculates stopping powers (the amount of energy lost per unit distance for both radiative and collisional events), energy deposits due to individual interaction types, and "real" pathlength (cumulative distance between events) which can be used to calculate detour factors (the ratio to the real range compared to the CSDA range).

\section{DISCUSSION}

Single scatter electron transport is time consuming. This method is not intended to be a general-purpose means of electron transport, but rather an extremely powerful tool for use in situations where it is desirable to obtain information about the basic interaction of electrons with the medium. Some timings are shown in Table I. In general, the simulation time increases with the number of histories, the geometry size, and as 

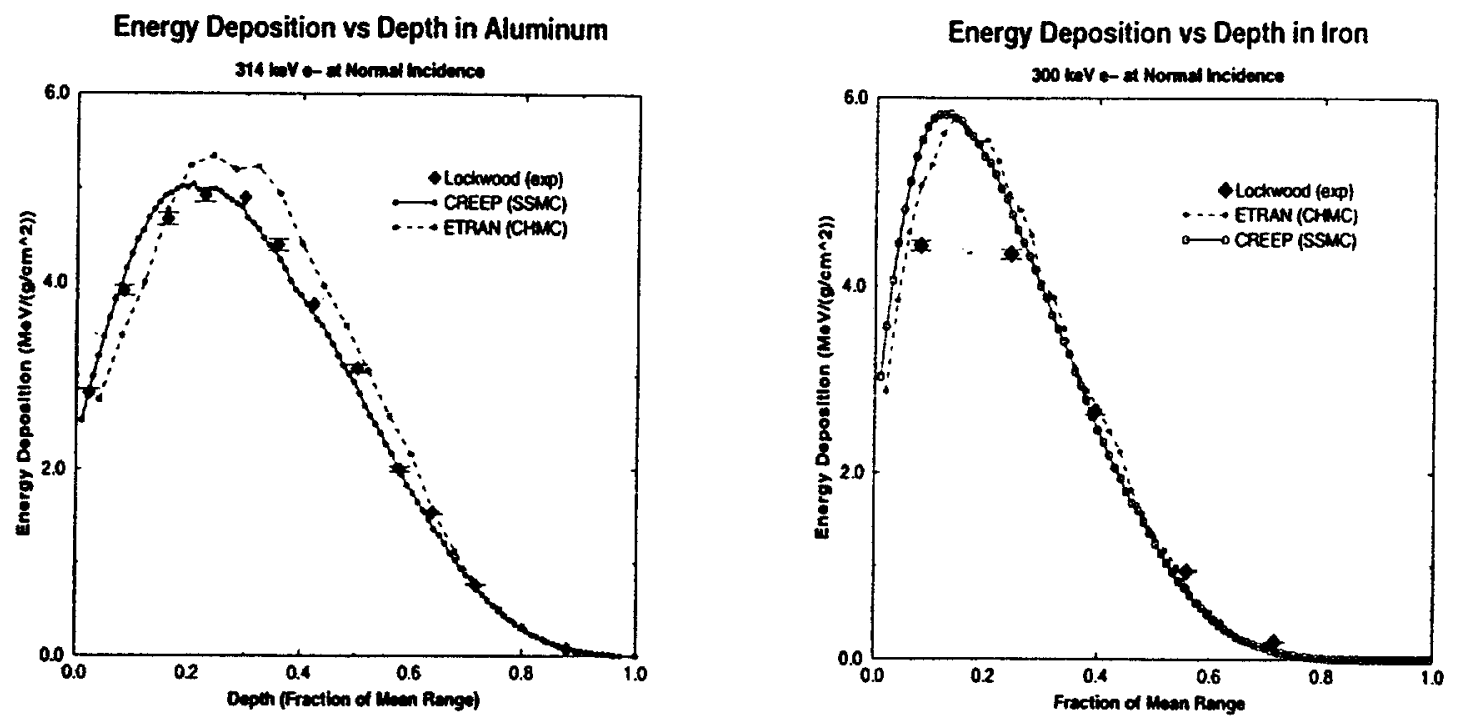

Figure 3: Energy deposition is shown as a function of depth into the medium, where the depth has been normalized to the CSDA range of the electron in each case. The points attributed to Lockwood et alare from calorimetric measurement; the comparisons are absolute [9]. These are compared to CREEP (single scatter Monte Carlo), as well ETRAN (condensed history Monte Carlo) as published in Lockwood [9].

the energy threshold is lowered. All of these require more interactions to simulated. The version of the code which includes compounds and mixtures is also notably slower than the element versions, due to the need to find cross sections in each element for every step, and then compare them to decide in which element the interaction will take place. Table 1 gives some feel for how the run time scales with different media.

Clearly if this method is to become practical, there must be a means for a radical speed up in the execution time. Such a means has been suggested in the Local-to-Global algorithm. [10] The Local-To-Global concept relies on tallying precomputed probability distributions resulting from a geometry element of fixed size, for a fixed incident energy and material type. Research in this area for CREEP is currently underway and is expected to lead to speed-ups which will lead to getting single scatter accuracy in about the same running time as is required by condensed history.

\section{CONCLUSIONS}

The need for a reliable low-energy electron transport method is currently great, and continues to grow with advances in microdosimetry and microtechnology, especially given the fact that some condensed history approximations tend to become poor at low energies and/or small step sizes [4]. Can SSMC fill this need?

The answer of course depends on the context. Although there are no inherent step size limitations, at energies comparable to the binding energy of the material, there are at least three approximations in CREEP that become weak, as pointed out earlier. First, the kinematics which are used to find the scatter angle after an ionization event neglect binding energy. This alone will probably not cause a major perturbation in the electron trajectory, since large-angle electron scatter is primarily an elastic event. Second, in compounds, chemical binding effects are neglected. So, for example, the binding energy of an $\mathrm{H}_{2}$ molecule is $16.4 \mathrm{eV}$, but CREEP would use the binding energy of elemental hydrogen, $13.6 \mathrm{eV}$. Third, as mentioned previously, the binding energy is locally deposited, rather than simulating the atomic relaxation process and tracking 
Table 1: Timings for several CREEP runs on a SunSparc 20 running Solaris OS 2.51. Each medium was a slab of $1 \mathrm{~mm}$ thick. Results for the number of interactions, the number of calls to the random number generator (RNG), and the user time are normalized per incident history.

\begin{tabular}{c|c|c|c|c|c} 
Medium & Density $(\mathrm{g} / \mathrm{cc})$ & Interactions & RNG calls & User Time (s) & Ratio \\
\hline \hline $\mathrm{H}$ & $1 \times 10^{-4}$ & 0.667 & 8 & $2.52 \times 10^{-4}$ & 1 \\
\hline $\mathrm{O}$ & $1.4 \times 10^{-3}$ & 5.098 & 39 & $1.77 \times 10^{-3}$ & 7.02 \\
\hline $\mathrm{Na}$ & 1.0 & 6714 & 48001 & 1.35 & 5357 \\
\hline $\mathrm{H}_{2} \mathrm{O}$ & 1.0 & 8057 & 71714 & 3.30 & 13095 \\
\hline $\mathrm{Au}$ & 19.3 & 19810 & 145541 & 6.98 & 27698 \\
\hline
\end{tabular}

the resultant Auger electrons and fluorescent photons. Although LLNL maintains a database that would address these issues (EADL), it has not been coupled to CREEP's transport. In addition, the non-discrete treatment of excitation events may also present problems at these energies or higher regimes if the spatial extent of the medium is on the order of the number of pathlengths required to traverse it. A simple energy straggling algorithm for excitation would probably soften these effects, however. Finally, and most fundamentally, there is the problem of the uncertainity of the cross section data itself.

Because of its slow run times, the single scatter algorithm presented here is not a general-purpose transport tool. However, there are certain instances in which it is desirable to obtain the most accurate answer possible - regardless of the time required. When does single scatter simulation have the most acceptable run times? It is most feasible when the total number of interactions is relatively small, as in a case involving low initial energy, a thin target, minute volume, or sparse media. Chemically simple media are much faster than media which have many elemental components.

Eventually it is expected that SSMC may become useful as a benchmarking tool for new scattering theories, a learning tool for developing physical intuition about parts of a large problem, and a modeling tool for small instruments such as some ion chambers, and other devices for studying energy deposition or interaction sites on a small scale.

\section{ACKNOWLEDGMENTS}

The author would like to thank James Rathkopf, Alex Bielajew, T. Rock Mackie, William Chandler, and D.E. Cullen for general advice and many helpful discussions. This work has been performed under the auspices of the U.S. Department of Energy by the Lawrence Livermore National Laboratory under contract number W-7405-ENG-48. 


\section{References}

[1] D.E. Cullen and S.T. Perkins. The Livermore Bremsstrahlung Database. UCID-21627, 1989. Lawrence Livermore National Laboratory.

[2] S.T. Perkins and D.E. Cullen. The Livermore Electron Impact Ionization Database. UCID-21628, 1989. Lawrence Livermore National Laboratory.

[3] S.T. Perkins and D.E. Cullen. The Livermore Electron Elastic Scattering Database. UCRL-ID-103170, 1990. Lawrence Livermore National Laboratory.

[4] C.T. Ballinger, D.E. Cullen, S.T. Perkins, J.A. Rathkopf, W.R. Martin, and S.J. Wilderman. Singlescatter Monte Carlo compared to condensed history results for low energy electrons. Nuclear Instruments and Methods in Physics Research B, pages 19-27, 1992.

[5] L.I. Schiff. Energy-Angle Distribution of Thin Target Bremsstrahlung. Physical Review, 83(2):252-3, 1951.

[6] E.H. Darlington. Backscattering of $10-100 \mathrm{keV}$ electrons from thick targets. J. Phys. D Appl. Phys, 8:85-93, 1975.

[7] G. Neubert and S. Rogaschewski. Backscattering Coefficient Measurements of 15 to $60 \mathrm{keV}$ electrons for Solids at various angles of incidence. Phys. Stat. Sol., 59:35-41, 1980.

[8] L. Landau. On the Energy Loss of Fast Particles by Ionization. Journal of Physics USSR. 8(4):201-5, 1944.

[9] G.J. Lockwood, L.E. Ruggles, G.H. Miller, and J.A. Halbleib. Calorimetric Measurement of Electron Energy Deposition in Extended Media - Theory vs Experiment. Sandia Report SAND79-0414, 1980.

[10] M.M. Svatos, C.T. Ballinger, H. Neuenschwander, T.R. Mackie, W.P. Chandler, C.L. Hartmann Siantar, J.A. Rathkopf, and P.J. Reckwerdt. Electron Transport in Radiotherapy using Local-to-Global Monte Carlo. In Preceedings of the International Conference on Mathematics and Computations. Reactor Physics, and Environmental Analyses, pages 866-875, La Grange Park, IL, 1995. American Nuclear Society, Inc.

[11] M.J. Berger. Monte Carlo calculation of the penetration and diffusion of fast charged particles. Methods in Computational Physics, 1:135-215, 1963.

[12] G. Moliere. Theorie der Streuung schneller geladener. Naturforsch, 2a:133, 1947.

[13] S. Goudsmit and J. L. Saunderson. Multiple Scattering of Electrons. Physical Review, 57:24-29, jan 1940.

[14] M.J. Berger and S.M. Seltzer. Bremsstrahlung and photoneutrons from thick tungsten and tantalum targets. Physical Review, C(2):621-631, 1970.

[15] J. Halbleib. Structure and Operations of the ITS Code System. In Theodore M. Jenkins, Walter Nelson, and Alessandro Rindi, editors, Monte Carlo Transport of Electrons and Photons, New York and London, 1988. Plenum Press.

[16] J.F. Briemeister. MCNP4 - A General Monte Carlo Code for Neutron, Photon, and Electron Transport. In LA-7936-M Rev. 4, Los Alamos National Laboratory, 1991.

[17] A. Bielajew. Plural and multiple small-angle scattering from a screened Rutherford cross section. Nuclear Instruments and Methods, B86:257-269, 1994. 


\section{APPENDIX - Condensed History Monte Carlo}

The technique of condensed history [11] is the most prevalent type of electron transport. It circumvents the problem of too many interactions by "condensing" the effect of numerous collisions into a single "step" through the medium. Rather than attempting to model all interactions, a representation of the aggregate effect of multiple collisions after a given step size is made.

In traditional condensed history methods, there are three categories of approximations made concerning the net effect of many interactions on the energy loss and spatial positioning of the electron. A multiscatter angular deflection distribution (usually that of Moliere [12] or Goudsmit-Saunderson [13]) is used to select the scattering angle at the end of a step. A straight-line approximation, modified by a correction to take intc account that the electron is not exactly traveling in a straight path during the step, is included. Finally, an expression for energy loss during a step is also required, which should account for the statistical variation in the energy deposited for a given distance traveled.

Condensed history codes are further be broken down into class I and class II algorithms. In class I algorithms, such as ETRAN [14], ITS [15], and MCNP [16], there are no individual collisions modeled; multi-scatter distributions are used exclusively. In class II algorithms, such as EGS4, certain "catastrophic" interactions are modeled individually, while the rest are handled with restricted multi-scattering distributions.

Both classes of condensed history methods have a limited range of validity as a result of relying on empirical approximations. For example, Ballinger has shown [4] that at low energies (below a few keV) the results of class I condensed history codes are inaccurate. This is partially because they rely on a Landau distribution [8] for energy loss, which allows the possibility of large energy losses, but deposits the energy locally instead of transferring it to secondary electrons. It is thus not reliable for situations involving secondary disequilibrium. Class II electron transport codes explicitly transport secondary electrons above a threshold energy, but they also break down at low energies. This is due in part to the small sub-step sizes that are used at low energies; very small sub-steps do not contain enough interactions to make the (Moliere) multi-scatter distributions valid. ${ }^{2}$ Short sub-steps not only cause the simulation to slow down, they often cause inaccurate electron energy loss. In both class I and class II algorithms, large sub-steps increase the efficiency, but the results can be inaccurate due to poor representation of the electron location, since the straight flight path approximation becomes worse as the step size increases.

\footnotetext{
${ }^{2}$ An exception is Bielajew's recent single-scatter extension of the Moliere theory for EGS4 [17].
} 


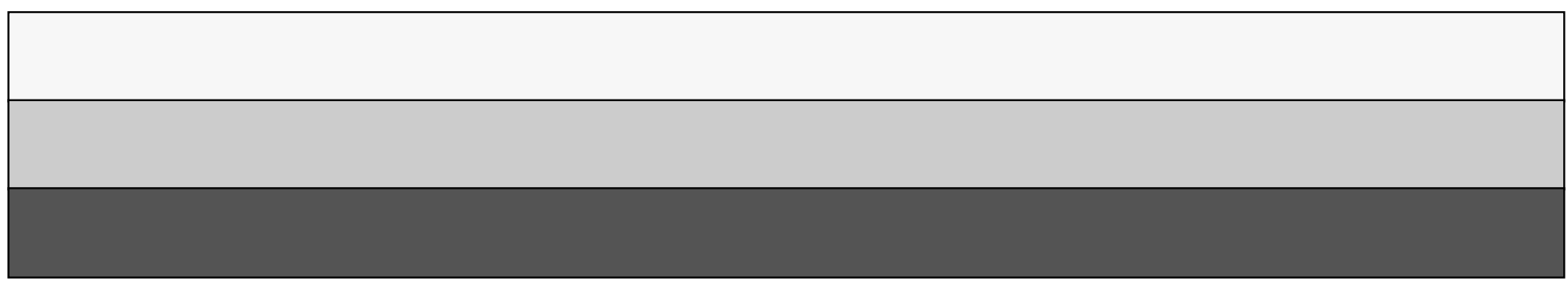

